\title{
Laparoscopic Ovarian Drilling in Clomiphene-resistant Polycystic Ovarian Syndrome Women: A Retrospective Analysis
}

\author{
${ }^{1}$ Sumi Thomas, ${ }^{2}$ Vivek P Vithayathil, ${ }^{3}$ Achamma Chandy, ${ }^{4}$ Thaiparambil K Aleyamma, ${ }^{5}$ Mohan S Kamath
}

\begin{abstract}
Introduction: Ovulation induction in women with polycystic ovarian syndrome (PCOS) can be carried out with drugs, such as clomiphene citrate (CC), which remains the first-line treatment option, and surgery, such as laparoscopic ovarian drilling (LOD), which is usually recommended as one of the second-line treatment options. Laparoscopic ovarian drilling may avoid or reduce the need for gonadotropins and at the same time reduce ovarian hyperstimulation syndrome (OHSS) and multiple pregnancy.
\end{abstract}

Aim: We aimed to evaluate the effectiveness of LOD in clomiphene-resistant women and to identify the clinical factors that might predict its success.

Study design: Retrospective analysis in a tertiary level infertility unit.

Materials and methods: All CC-resistant PCOS women who underwent LOD over a 10-year period were included. Polycystic ovarian syndrome was defined as per the Rotterdam criteria. The follow-up period was up to 3 years post-LOD. Outcomes, such as onset of regular cycles, spontaneous pregnancies, and live births were recorded.

Results: A total of 59 PCOS women who underwent LOD and were available for follow-up were included in the study. Majority of the patients were less than 30 years of age with a body mass index $(\mathrm{BMI})>24 \mathrm{~kg} / \mathrm{m}^{2}$. Out of those, 12 conceived $(20.3 \%)$ spontaneously and $14(23.7 \%)$ had regular menstrual cycles after the procedure. The live birth rate was $16.9 \%(10 / 59)$. We did not find any association of clinical factors, such as age, BMI, type of infertility, and history of irregular cycles with LOD success.

Conclusion: Laparoscopic ovarian drilling can be offered as a treatment option in select group of CC-resistant women with reasonable success. Post-LOD, for those who do not have resumption of regular cycles, alternate method of ovulation induction can be initiated.

Keywords: Clomiphene resistance, Laparoscopic ovarian drilling, Polycystic ovarian syndrome.

\footnotetext{
${ }^{1}$ Assistant Professor, ${ }^{2}$ Consultant, ${ }^{3}$ Assistant Physician ${ }^{4,5}$ Professor

1,3-5 Department of Reproductive Medicine, Christian Medical College, Vellore, Tamil Nadu, India

${ }^{2}$ Department of Reproductive Medicine, PRS Hospital Thiruvananthapuram, Kerala, India

Corresponding Author: Sumi Thomas, Assistant Professor Department of Reproductive Medicine, Christian Medical College, Vellore, Tamil Nadu, India, Phone: +914162283301 e-mail: drsumithomas@gmail.com
}

How to cite this article: Thomas S, Vithayathil VP, Chandy A, Aleyamma TK, Kamath MS. Laparoscopic Ovarian Drilling in Clomiphene-resistant Polycystic Ovarian Syndrome Women: A Retrospective Analysis. Int J Infertil Fetal Med 2017;8(3):93-96.

Source of support: Nil

Conflict of interest: None

Date of received: 02/07/2017

Date of acceptance: 29/08/2017

Date of publication: November 2017

\section{INTRODUCTION}

Polycystic ovarian syndrome is one of the commonest causes of anovulatory dysfunction. The prevalence of PCOS varies according to the different diagnostic criteria used for diagnosis, varying between $6 \%$ (National Institutes of Health criteria) and $15 \%$ when the broader Rotterdam criterion is applied. ${ }^{1}$ Among the PCOS women, further phenotypes have been suggested, such as complete, normoandrogenic, nonpolycystic ovaries, and ovulatory phenotype. ${ }^{2}$

The recommended first-line pharmacological treatment option for PCOS patients with anovulatory dysfunction is ovulation induction with CC. ${ }^{3}$ Women with unsuccessful clomiphene therapy mainly belong to two groups: CC failure (those who ovulated while on clomiphene but did not conceive) and CC resistance (failure to ovulate with $150 \mathrm{mg}$ of CC). Women who do not conceive following CC are usually offered other antiestrogen agents (tamoxifen), aromatase inhibitors (anastrozole), insulin-sensitizing drugs, exogenous gonadotropins, and, in some cases, LOD. ${ }^{4}$ Laparoscopic ovarian drilling has replaced the more invasive ovarian wedge resection as a surgical treatment option for PCOS women with anovulatory infertility. ${ }^{5}$ In contemporary practice, LOD is mainly indicated for CC resistance women who constitute approximately 20 to $25 \%$ of PCOS women undergoing CC therapy. ${ }^{4}$ The actual mechanism of how LOD works has not been fully elucidated. It is hypothesized that LOD destroys the ovarian androgenproducing tissue and, hence, reduces the peripheral conversion of androgens to estrogens. ${ }^{5} \mathrm{~A}$ fall in the serum levels of androgens and luteinizing hormone (LH) and 
an increase in follicle-stimulating hormone (FSH) levels have been demonstrated after ovarian drilling, thereby converting the androgen-dominant intrafollicular environment to an estrogen-rich milieu. ${ }^{6,7}$ Thus, both local and systemic effects are thought to promote follicular recruitment, maturation, and subsequent ovulation.

The gonadotropin therapy though effective requires careful monitoring with attendant risk of OHSS and multiple pregnancies. Women may need up to 3 to 6 treatment cycles of gonadotropin induction. Similar live births rates have been obtained following LOD compared with other medical options in CC-resistant women. The main advantage of LOD is significantly lower multiple pregnancy rates compared with gonadotropin therapy. The cost per live birth also has been shown to be lower following LOD compared with gonadotropin induction. ${ }^{5}$ Laparoscopic ovarian drilling, however, has been found to be beneficial for only a particular subset of PCOS women. Studies have suggested predictive factors of success of LOD, such as women with low BMI, shorter duration of infertility, and younger age. ${ }^{8}$ We decided to evaluate the effectiveness of LOD in clomiphene-resistant women and also to identify the clinical factors which might predict its success.

\section{MATERIALS AND METHODS}

We conducted a retrospective analysis of all the PCOS women who underwent LOD over a 10-year period between February 2003 and February 2013. All the women were diagnosed with PCOS according to Rotterdam criteria. ${ }^{9}$ As per the unit protocol, the treatment-naïve women were advised CC for ovulation induction. The starting dose was $100 \mathrm{mg}$ per day for 5 days and in case of lack of follicular development, the dose was increased to a maximum of $200 \mathrm{mg}$ per day. If the women did not have follicular development with $200 \mathrm{mg}$ dosage, they were diagnosed as CC resistant.

Clomiphene citrate-resistant women were given the option of insulin-sensitizing therapy, gonadotropin induction, or LOD. A written informed consent was taken from women who decided on LOD as a treatment option.

Laparoscopic ovarian drilling was performed under general anesthesia. After introducing the laparoscope, the pelvic organs were carefully inspected for any other pelvic pathology, such as endometriosis and tubal adhesions. For LOD, the ovary was stabilized and Corson needle (Karl Storz, Tuttlingen, Germany) was used to pierce the ovarian cortex using a monopolar diathermy electrosurgical unit. The current was set at $30 \mathrm{~W}$. Cutting mode was used to penetrate the ovarian cortex and coagulation mode was used when the needle electrode was in the ovarian stroma. The needle electrode was pressed against the antimesenteric border of the ovary for approximately 5 seconds to achieve penetration of the ovarian capsule to a depth of $8 \mathrm{~mm}$. Each ovary was punctured at four points. ${ }^{6}$ Adequate care was taken to avoid vascular hilum. At the completion of the procedure, the ovarian surface was washed with saline to bring down the ovarian temperature.

Postoperatively, the women were advised to maintain menstrual calendar and try to conceive naturally. Initially, they were reviewed in the outpatient department after 3 months and subsequently after 6 to 12 months. For women whose menstrual cycles did not regularize after 6 to 12 months and for older women with longer duration of infertility, insulin sensitizers or gonadotropin induction was offered. If none of these options were successful, assisted reproductive technology was advised as the final treatment option.

The patient's medical records were obtained in medical records section and data were collected. The follow-up period was up to 3 years postprocedure. In case of any missing data, the patients were contacted by phone or e-mail.

Data were analyzed using Statistical Package for the Social Sciences version 11 (SPSS Inc., Chicago, Illinois, USA).

\section{RESULTS}

There were a total of 59 patients with clomiphene resistance who underwent LOD and were available for follow-up during the study period. A total of 52 (88\%) women had primary infertility and seven women (12\%) had secondary infertility. Majority of patients were in the younger age group of 25 to 30 years (83\%) and had BMI of more than $24 \mathrm{~kg} / \mathrm{m}^{2}$ (63\%). Spontaneous regular cycles resumed in $23.7 \%$ patients (14/59) at the 3-month follow-up period.

A total of 12 patients conceived spontaneously out of $59(20.3 \%)$ patients within the follow-up period of 3 years. A total of 10 women had live birth (16.9\%) and other $2(3.3 \%)$ had miscarriage. Among these 12 patients, 9 (75\%) women conceived within 1 year of the procedure.

We did not find any significant difference between women who conceived and those who did not conceive with regard to clinical factors, such as age (less or more than 30 years), BMI (less or more than $24 \mathrm{~kg} / \mathrm{m}^{2}$ ), duration of infertility (less or more than 3 years), primary or secondary infertility, and prior menstrual cycles (anovulation or oligo-ovulation) (Table 1). Spontaneous resumption of regular cycles was associated with a higher pregnancy rate [64.28\% (9/14) vs 6.6\% (3/45); $\mathrm{p}=0.0001]$. There were no multiple pregnancies or surgical complications following LOD. Out of the remaining 47 patients who were followed up, 19 (40.4\%) patients conceived with 
LOD in Clomiphene-resistant PCOS Women

Table 1: Clinical factors and its association with post-LOD pregnancy rate

\begin{tabular}{|c|c|c|c|}
\hline Parameters & $\begin{array}{l}\text { Laparoscopic } \\
\text { ovarian drilling } \\
\text { spontaneous } \\
\text { conception } \\
(n=12)\end{array}$ & $\begin{array}{l}\text { Laparoscopic } \\
\text { ovarian drilling no } \\
\text { conception in } \\
3 \text { years }(n=47)\end{array}$ & $p$-value \\
\hline \multicolumn{4}{|l|}{ Age (years) } \\
\hline$\leq 30$ & 11 & 38 & 0.6 \\
\hline$>30$ & 1 & 9 & \\
\hline \multicolumn{4}{|l|}{$B M I\left(k g / m^{2}\right)$} \\
\hline$\leq 24$ & 6 & 16 & 0.3 \\
\hline$>24$ & 6 & 31 & \\
\hline \multicolumn{4}{|c|}{$\begin{array}{l}\text { Duration of } \\
\text { infertility (years) }\end{array}$} \\
\hline$\leq 3$ & 7 & 19 & 0.3 \\
\hline$>3$ & 5 & 28 & \\
\hline \multicolumn{4}{|l|}{ Infertility } \\
\hline Primary & 12 & 40 & 0.3 \\
\hline Secondary & 0 & 7 & \\
\hline
\end{tabular}

other treatment modalities after LOD within the study period (Table 2).

\section{DISCUSSION}

In our study, approximately one in six CC-resistant women $(16.9 \%)$ had a live birth after LOD within the follow-up period of up to 3 years. A total of 12 women had conceived (20.3\%) but two pregnancies ended up in a miscarriage. Resumption of spontaneous menstruation was noted in 14 patients $(23.7 \%)$ and was found to be associated with significantly higher pregnancy rates compared with those who continued to have irregular menstrual cycles ( 64.28 vs $6.6 \%$; $p=0.0001$ ).

Randomized trials have reported pregnancy rates of 25 to $51 \%$ and live birth rates of 24 to $44 \%$ following LOD in PCOS women. ${ }^{5}$ Other nonrandomized follow-up studies have obtained higher pregnancy rates of up to 45 to $60 \%$ following LOD. ${ }^{10-12}$ We found the live birth and pregnancy rates of 16.9 and $20.3 \%$ respectively, which are lower compared with earlier studies. This disparity could be due to the heterogeneity in population studied, different techniques employed, and varying period of follow-up. Worldwide, it has been observed that in PCOS women, the prevalence of metabolic syndrome varies according to ethnic and regional factors, with rates varying from $1.6 \%$ in Czech women to $37 \%$ in Indian women and $39.5 \%$ in Iranian population. ${ }^{13,14}$ Similarly, PCOS women who are included in studies consist of varying mix of different PCOS phenotypes, with some study population consisting of higher proportion of more resistant forms (hyperandrogenic) which may reduce the effectiveness of LOD. This, along with the fact that we included only CC-resistant women, may be the probable reason for lower pregnancy rate in our study.
Table 2: Post-LOD conceptions following alternate methods of ovulation induction

\begin{tabular}{ll}
\hline $\begin{array}{l}\text { Method of assistance following } \\
\text { laparoscopic ovarian drilling }\end{array}$ & $\begin{array}{l}\text { Number of patients who } \\
\text { conceived }(n=19)\end{array}$ \\
\hline Clomiphene & 2 \\
\hline Gonadotropin with IUI & 6 \\
Gonadotropin stimulation & 7 \\
In vitro fertilization & 4 \\
\hline
\end{tabular}

IUI: Intrauterine insemination

Resumption of spontaneous cycles has been found in up to 55 to $90 \%$ of PCOS women following LOD. ${ }^{12,15}$ We found spontaneous regular periods in $23.7 \%$ women, which are lower when compared with findings of earlier studies. However, we found significantly higher pregnancy rates in those who had resumption of regular cycles compared with those who continued to have irregular cycles (64.3 vs 6.6\%; $\mathrm{p}=0.0001$ ). This is mostly explained by the onset of ovulatory cycles as suggested by regularization of cycles and can help clinicians decide regarding the need to proceed for alternative ovulation induction methods in women with persistent irregular cycles post-LOD.

Hashim ${ }^{16}$ studied the predictors for success of LOD and found women with obesity $\left(>25 \mathrm{~kg} / \mathrm{m}^{2}\right)$, low basal LH levels (10 IU/1), longer duration of infertility (>3 years), and marked hyperandrogenism responded poorly to LOD. Kaur et $\mathrm{al}^{10}$ showed that CC-resistant PCOS women who had a higher LH:FSH ratio prior to LOD had a poorer pregnancy rate compared with those who had a normal LH:FSH ratio. We found no clinical factors that could predict success of LOD and help prognosticate preoperatively. Woman's age (less and more than 30 years), BMI (less and more than $24 \mathrm{~kg} / \mathrm{m}^{2}$ ), duration of infertility (less and more than 3 years), menstrual irregularity (oligo-ovulation or anovulation), and type of infertility (primary and secondary) were not found to be associated with probability of success after LOD. We did find that the postoperative resumption of regular cycles was significantly associated with higher pregnancy chances.

Time to pregnancy is an important factor from patients' perspective. Most of the recent studies have shown a successful outcome within 3 to 6 months following LOD and have resorted to other modalities of ovulation induction in case of oligo-ovulation or nonresumption of spontaneous cycles. In our study, though we followed up the patients for 3 years post-LOD, majority (75\%) of the women conceived spontaneously within 1 year of the procedure and 3 months following LOD if there was nonresumption of spontaneous cycle, ovulation induction with medications was initiated as the anxious couple may not wish to wait longer. 
Small sample size and retrospective nature of the study are some of the important limitations of our study.

\section{CONCLUSION}

In certain select group of CC-resistant PCOS women, who may not be able to undergo repeated gonadotropin induction, LOD can be offered as a one-time intervention especially when tubal evaluation is also warranted. PostLOD, for those who do not have resumption of regular cycles, alternate method of ovulation induction can be initiated.

\section{REFERENCES}

1. Fauser B, Tarlatzis BC, Rebar RW, Legro RS, Balen AH, Lobo R, Carmina E, Chang J, Yildiz BO, Laven JS. Consensus on women's health aspects of polycystic ovary syndrome (PCOS): the Amsterdam ESHRE/ASRM-Sponsored 3rd PCOS. Consensus Workshop Group. Fertil Steril 2012 Jan;97(1):28-38.

2. Kar S. Anthropometric, clinical, and metabolic comparisons of the four Rotterdam PCOS phenotypes: a prospective study of PCOS women. Hum Reprod Sci 2013 Jul-Sept;6(3):194-200.

3. Use of clomiphene citrate in infertile women: a committee opinion. The Practice Committee of the American Society for Reproductive Medicine. Fertil Steril 2013 Aug;100(2):341-348.

4. Kaur S, Saha L. Medical management of clomiphene-resistant polycystic ovarian syndrome: an update. Int J Basic Clin Pharmacol 2014 Jan-Feb;3(1):1-9.

5. Farquhar C, Brown J, Marjoribanks J. Laparoscopic drilling by diathermy or laser for ovulation induction in anovulatory polycystic ovary syndrome. Cochrane Database Syst Rev 2012 June;13(6):CD001122.

6. Mitra S, Nayak PK, Agrawal S. Laparoscopic ovarian drilling: an alternative but not the ultimate in the management of polycystic ovary syndrome. J Nat Sci Biol Med 2015 Jan-June;6(1):40-48.

7. Flyckt RL, Goldberg JM. Laparoscopic ovarian drilling for clomiphene-resistant polycystic ovary syndrome. Semin Reprod Med 2011 Mar;29(2):138-146.
8. Baghdadi LR, Hashim HA, Amer SAK, Palomba S, Falbo A, Al-Ojaimi E, Ott J, Zhu W, Fernandez H, Nasr A, et al. Impact of obesity on reproductive outcomes after ovarian ablative therapy in PCOS: a collaborative meta-analysis. Reprod Biomed Online 2012 Sep;25(3):227-241.

9. Consensus on infertility treatment related to polycystic ovary syndrome. The Thessaloniki ESHRE/ASRM-Sponsored PCOS Consensus Workshop Group March 2-3, 2007, Thessaloniki, Greece. Hum Reprod 2008 Mar;23(3):462-477.

10. Kaur M, Pranesh G, Mittal M, Gahlan A, Deepika K, Shashikala T, Rao KA. Outcome of laparoscopic ovarian drilling in patients of clomiphene resistant polycystic ovarian syndrome in a tertiary care center. Int J Infertil Fetal Med 2013 May;4(2):39-44.

11. Nasr AA, El-Naser A, El-Gaber AA, El-Saar MA, Mgeed AA, Abolfotouh MM. A modified technique of laparoscopic ovarian drilling for polycystic ovary syndrome using harmonic scalpel. J Diabetes Metab. 2012 Oct;30;56:008.

12. El Sharkwy IA. Metformin versus laparoscopic unilateral ovarian drilling in clomiphene resistant women with polycystic ovary syndrome. Middle East Fertil Soc J 2013 Sept;18(3): 202-207.

13. Fatemeh Pourteymour Fard Tabrizi, Beitollah Alipoor, Mahzad Mehrzad Sadaghiani, Alireza Ostadrahimi, Aida Malek Mahdavi. Metabolic syndrome and its characteristics among reproductive-aged women with polycystic ovary syndrome: a cross-sectional study in Northwest Iran. Int J Fertil Steril 2013 Jan-Mar;6(4):244-249.

14. Mandrelle K, Kamath MS, Bondu DJ, Chandy A, Aleyamma TK, George K. Prevalence of metabolic syndrome in women with polycystic ovary syndrome attending an infertility clinic in a tertiary care hospital in South India. J Hum Reprod 2012 Jul;5(1):26-31.

15. Elsamy E, Saleh S. Impact of laparoscopic ovarian drilling on hormonal profile and clinical features in women with polycystic ovary syndrome. Int J Gynecol Obstetrics 2015;20(1):1-5.

16. Hashim HA. Predictors of success of laparoscopic ovarian drilling in women with polycystic ovary syndrome: an evidence-based approach. Arch Gynecol Obstet 2015 Jan;291(1):11-18. 